\title{
AC 2010-104: EXTRACTING EXPERT KNOWLEDGE ON GEOTECHNICAL FAILURES FOR USE IN CIVIL ENGINEERING EDUCATION
}

\section{Luis Godoy, University of Puerto Rico}

Prof. Luis A. Godoy graduated as a Civil Engineer at the National University of Córdoba, Argentina, and received his Ph. D. from University College London (University of London, UK) in 1979. He is Professor at the University of Puerto Rico at Mayagüez since 1994. Godoy has published three books: Thin Walled Structures with Structural Imperfections (Pergamon Press, 1996), and Theory of Elastic Stability (Taylor and Francis, 2000), and Stability of Structures: A historical perspective (CIMNE, 2009). His research interests include engineering education, structural stability, granular flow, computational mechanics, and plates and shells, and results of his research have been published in more than 150 peer-reviewed journal papers. At present, he has been awarded an NSF-CCLI research grant.

\section{Pedro Covassi, National University of Cordoba (Argentina)}

Pedro A. Covassi graduated as a Civil Engineer at the National University of Cordoba (Argentina) and is currently a doctoral student at the National University of Cordoba in Argentina working in the Geotechnical Laboratory. He has been awarded a scholarship from the National Agency for the Promotion of Science and Technology (FONCYT) in Argentina. 


\title{
Extracting Expert Knowledge on Geotechnical Failures for Use in Civil Engineering Education
}

\begin{abstract}
The work reported in this paper is part of a broader research aimed at developing computer-based tools to teach engineering failures to civil engineering students. To identify what topics and procedures should be taught to engineering students to help them work in the field of analysis of geotechnical failures, interviews were conducted to expert engineers, with emphasis on their methodology of research, their approach to the construction of failure hypothesis, and their reasoning about causes of failure. Seven experts with ample experience in investigating geotechnical failures were interviewed using a structured, face-to-face questionnaire. The list of questions was divided into: (a) General questions about the relation between the expert and his/her work; (b) Questions about a specific case in which the expert was involved; (c) Questions about the methodology used in this investigation; and (d) Questions about the development of failure hypothesis in this case. Open questions were formulated to stimulate having a free discussion about the question domain. An analysis of the interviews indicates that experts use analogy to establish relations between the case in hand and other cases; this may or may not be useful in terms of solving a failure problem, but it becomes part of the knowledge stored by the expert.
\end{abstract}

Keywords: case-based reasoning, experts and novices, interviews, geotechnical failures.

\section{Introduction}

This paper reports research carried out in order to identify what knowledge and procedures should be taught to undergraduate civil engineering students, and what kind of advice should be given to them, in order to help them work investigating geotechnical failures.

Interest in the work done by experts and novices in various fields of science and engineering emerged in the 1960s as an attempt to implement expert knowledge in artificial intelligence software. This goal of developing computer codes capable of understanding and solving problems was the initial driving force; however, an educational interest in this field emerged in the $1990 \mathrm{~s}^{2}$ under the assumption that understanding how experts solve problems would indicate what should be taught to students on their way to becoming experts. Ahmed et al. ${ }^{1}$ focused their work on how experts perform aeronautical engineering design, for which they examined ways in which experts and novices performed design tasks. Such studies are valuable because they emphasize what experts do while solving problems close to their natural environment.

A second approach in this field is the study of cases of historical interest, for which information is available and lessons can be learned. Petroski ${ }^{2}$ investigated failure cases to characterize and classify sources of engineering failures. Delatte ${ }^{3}$ compiled a collection of civil engineering failures in the form of case-studies, in an attempt to help students gain experience by considering cases investigated by others. The general framework for the study of cases is known as Case-Based Reasoning ${ }^{4}$.

A third avenue is the elicitation of expert knowledge by means of interviews. Knowledge acquisition is a crucial topic in knowledge-based engineering, and a common way to capture knowledge from experts is by means of interviews: "The main vehicle for knowledge elicitation is face-to-face discussions between the expert who possesses the domain 
knowledge and the knowledge engineer who asks questions, observes the expert solving problems, and determines what knowledge is being used" 5 (pp. 349).

This methodology based on interviews has been followed in this research in order to obtain information regarding how experts reason and proceed in the solution of engineering failure analysis. Specifically, this paper concentrates on interviews with geotechnical experts, whereas the responses of structural experts investigating failures have been addressed in a recent paper ${ }^{7}$.

Two main research hypotheses are considered in this paper: (1) Experts investigating geotechnical failures employ case-based reasoning and adapt solutions from old cases. In a more restricted formulation, this hypothesis states that experts investigate new cases by using their experience from previous cases and from what is reported in the technical literature. (2) Experts formulate a failure hypothesis as soon as the case is described to them and even before their first visit to the site of the failure.

\section{On Experts and Novices}

A review of the literature on experts and novices with an educational perspective was compiled by Bransford et al. ${ }^{7}$. Other authors, such as Vick ${ }^{8}$, considered civil engineering problems in order to understand expert knowledge. Those studies attempted to produce a synthesis of the differences between experts and novices. The main attributes of experts have been summarized as:

- Reasoning: Experts use forward reasoning (FR), whereas novices tend to use backward reasoning (BR) ${ }^{8}$. In FR, the information available on the specific case of interest is used in order to infer a solution; in BR, on the other hand, it is necessary to check all possible solutions, one by one, by comparing the available information and observe how it matches the case considered. Although BR may be efficient in computational intelligence environments, FR is used by human experts as a shortcut to solve problems using their previous knowledge.

- Level of understanding: Experts can identify the nature of a problem at a deep level and link the current problem with others by analogy; novices, on the other hand, are often limited to superficial aspects of a problem ${ }^{12}$. According to Bransford et al. ${ }^{7}$, "experts have acquired a great deal of content knowledge that is organized in ways that reflect a deep understanding of their subject matter".

- Storing: There are two main components in expertise: one is how much an experts knows, and a second component is how the expert accesses this knowledge. Experts can store knowledge in patterns or clusters rather than as disjointed pieces of information, so that retrieving information becomes more efficient. According to Bransford et al. 7 , "experts notice features and meaningful patterns of information that are not noticed by novices".

- Retrieving: Experts can visualize a problem and also its representation, so that they need to retrieve only the information that is relevant to the current case ${ }^{8}$. "Experts are able to flexibly retrieve important aspects of their knowledge with little attentional effort" 7 .

- Meta reflection: Experts are able to recognize the limitations of their conclusions ${ }^{8}$ : being an expert does not mean that a conviction of complete certainty is claimed. 


\section{Method: The Semi-structured Questionnaire}

A questionnaire was designed as part of this research to capture knowledge on how experts solve geotechnical failure investigations. Due to limitations in our access to experts, the questionnaire was formulated to be completed in one session (rather than having several sessions, each with a specific goal, as recommended in the literature ${ }^{5}$ ).

Because of this constraint, the interview contained two sets of questions in one condensed session. The first set was a kickoff part about some general experience of the expert. The aim was to understand general views of the experts regarding their field and what they considered to be more attractive and also more difficult. In the second set we asked the expert to recall one specific case and consider details of it. Emphasis was made to understand the context of each story told by an expert. This set, which took most of the time, contained the description of the case considered. The methodology employed was the subject of the third set, and the final set of questions referred to the reasoning used in solving the case.

There are two different problems that experts associate with geotechnical failures: the identification of the causes of failure and the solution proposed to restore the infrastructure or constructed facility to its original operating condition. Often the client is interested in the second problem and is only marginally interested in understanding the causes that produced a failure. Cause identification is highly relevant in cases of legal disputes regarding failure responsibility. Notice that the identification of causes is always an important component in structural failure analysis, as reported in ${ }^{6}$. No distinction was made in this questionnaire between the two problems, as they seem to be closely connected in the answers of experts.

The list of questions was divided into:

I. General questions about the relation between the expert and his/her work. This part was included in order to learn about the previous experience of the person interviewed. The specific questions in this group are:

I.1. What difficult geotechnical failure cases have you investigated yourself?

I.2. What aspects of that work do you like most?

I.3. What aspects are the most difficult part of the work?

II. Questions about a specific case in which the expert was involved. This part is important to focus on a single case and to make it as a case study.

II.1. Briefly describe a specific failure case in which you participated.

II.2. How were you contacted and who was the part interested in doing the investigation?

II.3. Why was the investigation necessary? What was the objective?

II.4. Were there any serious conflicts associated with this failure? Who were the stakeholders in the conflict?

II.5. Did you have access to all needed documents? Were there missing or unavailable documents when you requested them?

II.6. Were there previous studies about this failure, including the formulation of hypothesis? Did that influence your work?

II.7. Did you interview those who participated in the design and construction?

III. Questions about the methodology used in this investigation.

III.1. What was the sequence of activities that you followed in this investigation?

III.2. Did you visit the site after failure? Was access to the site easy? What was the benefit of the site visit?

III.3. Did you perform calculations and/or lab testing? What was the objective?

III.4. Were you able to find an explanation to the failure investigated? Describe briefly. 
IV. Questions about the development of a failure hypothesis in this case.

IV.1. Was this case similar to other cases in which you participated previously?

IV.2. Was this case similar to other cases that you found published in the technical literature?

IV.3. How did you formulate hypothesis about this failure?

IV.4. Did you have a hypothesis before visiting the site?

IV.5. What aspect of the investigation allowed you to think of a plausible hypothesis?

IV.6. Did you attempt to validate the hypothesis by testing or calculation?

IV.7. Were you satisfied with the validity of the hypothesis that you proposed? Did other peers provide you with feed-back or comments on them?

Questions are of open ended nature, to give the possibility of having a discussion about the question domain. The experts were told that they should not provide yes or no answers.

Seven face-to-face interviews were carried out during two months of research. All interviews were conducted by the second author. Experts were male practicing engineers, with either $\mathrm{Ph} . \mathrm{D}$. or P.E. A typical interview took one hour. After the interviews were conducted, we organized the answers by looking at common features in the responses.

We never had to handle problematic experts: They all understood the importance of the research and were very happy to cooperate. As Gonzalez and Dankel ${ }^{5}$ (pp. 352) state, "experts generally enjoy the opportunity to speak openly about their domain and show how much they know".

\section{Results: Responses to Selected Questions}

Because the main interest in this investigation was to understand how experts reason and formulate hypothesis, in this section we review only parts of the answers that are related to these interests.

Was this case similar to other cases in which you participated previously?

Expert A. "Not a landslide of this size. The maximum size that I had seen before was of 15 million cubic meters and this one was 1,000 million. I had never seen anything even similar to this case regarding size."

Expert B. "From the point of view of loss of frictional capacity, this case was similar to many other cases that I had seen before. From the point of view of the interaction with next door neighbor buildings, this was new to me."

Expert C. "The collapse of canals in collapsible soils is a recurrent problem, but the [remedial] solutions are all different. The solution I proposed in this case was what occurred to me at that time and is not a standard solution; it all depends on the client, the time of the year, and if it is possible to close the canal for a long time. If it is possible to close the canal for a year, the solution is different from a situation in which it is not possible to interrupt the water flow."

Expert D. "This case bears no relation with others in which I have been involved before." Expert E. "This case resembles two previous cases, but those were of smaller magnitude." Expert F. "Cases have always something similar, and this case was similar to others in..." Expert G: "Yes, at that time and in that geographical area, the problem was a subject of discussion because it was a generalized problem. Even us who worked in labs participated in those discussions." 
Common features:

- The experts had investigated failure cases of similar or related types, but not with the characteristics of the one they reported here. New features were present in the new case that were not present in other previous cases.

- Two experts identified significant differences in the size of the problem, which introduced differences in behavior.

Was this case similar to other cases that you found published in the technical literature?

Expert A. "There are not many cases of that size, which reach one cubic Km. But I had read about cases in which damming of several cubic Km were reported. However, most such failures were due to seismic movements. In order to mobilize such large mass, an important force is necessary [and this is generally provided by an earthquake]."

Expert B. "I had not read cases which were exactly the same as this one, but I had read about settlements and underpinnings. But the solution we proposed I had not seen being adopted in other places."

Expert C. "I have seen many times how this problem was solved in other places. This is a very common type of failure."

Expert D. "May be I had seen soothing before. In general terms, one can see many things and do not make a reference of it, but it remains at the back of our memory. I do not specifically recall (nor did I search) any specific bibliography, mainly because the mechanism was not complicated and all we had to do was to investigate the soil geotechnics and soil pressures." Expert E. "Yes, there were links with cases reported in journal papers."

Expert G: "No, there was little access to literature or to Internet, and we should have gone to a library if we wanted to read journals or books."

Common features:

- The existing literature was not the main source of direct information used by experts to identify causes or remedial solutions.

- Most investigators could not recall having seen a similar case in the literature.

- Existing information was useful only in indirect ways.

\section{How did you formulate a hypothesis about this failure?}

Expert A. "We formulated the hypothesis when we were at the field and noticed that not everything had moved. For that we had to walk the entire landslide from its base to the top. Geotechnical failures are usually associated with events that you have seen before. I believe that if one sees a failure of a slope, one attempts to see the same thing in a mental archive, including photographs, images or cases seen before. This is what I think an expert does: to accumulate information about a specific type of problem. If you are involved in a similar case, then you consider if it fits using analogy."

Expert B. "I formulated the hypothesis about a week after we made the reconnaissance of the failure. We considered the existing damage and then we thought of a hypothesis. We confirmed this using a numerical model; it took us a month to model the problem."

Expert C. "Only after observing and thinking. It was not done immediately. Sometimes one can have solutions in mind but they not always [adequate]. There are many ways to solve a problem, ranging from very expensive to very inexpensive solutions."

Expert D. "I formulated the hypothesis after the site visit. We assumed that we did not know if water had infiltrated through a crack or if the soil had fractured and the conditions had changed." 
Expert E. "I though about a failure hypothesis before the site visit, but could not verify it until model results were obtained."

Expert F. "The hypothesis was gradually formulated. This is not done immediately. It is not that I can say: 'now I propose a hypothesis'. Generally, I conceptualize the problem as I progress seeing it. Most likely during the first visit I have an idea of what happened. In this case I saw a landslide and generated a hypothesis, then went to a second site and attempted to compare both to establish if the variables that could have caused the problem matched or not. Then I observed the problem from a different perspective and had new thoughts. You leave the first visit with an idea, but in this case the hypothesis ended in its final form about two months after the first visit, and other aspects were confirmed during the second visit. Even today, other events continue occurring for which we do not have a clear explanation. It is a maturing process, at least in this case."

Expert G: "At the moment in which this failure occurred and based on previous information, one could formulate a hypothesis of what was happening."

Common features:

- In some cases, the expert could formulate a hypothesis during the first site visit.

- Most experts could formulate a hypothesis only after they visited the site, collected data and reconsidered the available evidence in his/her office.

- Computational models are not always considered necessary, but in some cases they are used to obtain a more complete picture of the situation.

\section{Did you have a hypothesis before visiting the site?}

Expert A. "As said before, we formulated a hypothesis in the field. We had the hypothesis of bending failure of the pipe before because they [the workers] had taken the pipe: it was bent and not broken. They noticed lower pressure values at the pumping and control stations." Expert B. "I did not have any hypothesis at the moment of the first visit. In cases like this one, the client often describes the symptoms but does not describe them well. Maybe it is not intentionally, but the client sees things differently from what we do."

Expert D. "In general, I go to the site and try to imagine the mechanisms, and those mechanisms you find as the visit progresses and data is gathered. Then we think of a hypothesis and identify the elements that should be verified. We take pictures and get recordings. We postulate what happened not during the visit but afterwards, while reviewing the photographs."

Expert E. "I had a hypothesis before the visit. I had even thought and worked in modeling the problem before the visit."

Expert F. "I had some preliminary ideas based on what had been told to me and the photographs that I received before the visit. But I did not have a hypothesis. Based on other cases that I had seen in other problems, I had an idea of what was happening. One goes to see the problem with an idea in mind, but this is modified as one becomes more involved in the case."

Expert G: "Yes, I had a hypothesis before visiting the site."

Common features:

- Experts formulate hypothesis during or after the first site visit.

- In cases in which information was sent to an expert before the site visit, he/she was able to formulate a preliminary hypothesis based on previous knowledge.

- Hypotheses provided by the client are often misguiding and loaded with their own expectations and needs. 
Expert A. "I do not think that this is about being sure of anything, but it is about getting an approximation. Maybe you never know the truth. Based on the knowledge [of the case] that we had, I think that the approximate explanation we made was reasonable. This was the only explanation for which we had real evidence. Anything else could not be verified."

Expert B. "At present I do not have any absolute certainty (as in everything else in our field). I believe that there was a combination of causes..."

Expert C. "I am sure that [the remedial] solution will work. There are no magic solutions..." Expert D. "I am completely sure about the hypothesis because soil pressures were computed again and we could verify that [the infrastructure] was poorly constructed."

Expert E. "The analysis we made had several limitations and did not take into account all variables or possible scenarios that could be considered. However, the solution is within the state of the art and professional practice."

Expert F. "This is nature, and there is no $100 \%$ certainty. I believe that many things that occur are considered [in an investigation] but others are not."

Expert G: "I am certain that this was the cause of failure."

Common features:

- Most experts were not completely sure about their work, but they understood that they reached a reasonable approximation based on the available evidence.

- Several experts recognized their own limitations concerning the identified cause or the proposed solution. This attribute was identified by Vick ${ }^{8}$ and was also an outcome of the present interviews.

- Certainty is sometimes claimed based on calculations carried out to confirm a hypothesis.

What aspects of the work do you like most?

Expert A. "In general terms, I am interested in jobs involving large failures, such as failures of considerable size... I am interested in problems in which size is an issue."

Expert B. "This work is usually divided in three parts; a problem of a geotechnical failure is a single problem, but usually the client is interested in understanding why this failure happened, what was the problem, and what is the solution that could be implemented. What I like the most are the relation with the client, the field work to collect evidence, and the analytical or experimental evaluation which follows."

Expert C. "The contract and the solution of the problem are not done based on the engineer's enjoyment: this is a professional work and you work under a contract with a client." Expert D. "I enjoy finding a cause and designing a methodology to find the cause." Expert E. "This is a challenge and allows one to learn from failures."

Expert F: "First, understanding the problem and then presenting a solution to the problem." Expert G: "Finding the cause."

Common features:

- Most experts enjoy "finding a failure cause". (Notice that the idea behind this is that a cause has been lost and should be found).

- Some experts enjoy the work, but only under certain conditions (such as being involved in a large failure investigation).

- One expert stressed the professional side of the work and questioned the idea of enjoyment associated with it. 
Expert A. "The most difficult part is the identification of the cause of failure. The most complicated part is not producing a model, but to imagine a failure mechanism, and what are the properties involved, which will be used to represent the problem in any analysis." Expert B. "The complexity of the field work depends on the experience of the investigator. Modeling is the most difficult part because problems have a systemic nature, data is usually insufficient, and one works with parameters that are considered to be most relevant. Often, parameters are uncertain and this introduces modeling difficulties."

Expert C. "Always the most difficult part is finding the cause or a solution."

Expert D. "In many geotechnical problems there is an assumption that a failure may have occurred but there are no visible signs. In such cases one needs to design a methodology to identify the failure itself."

Expert E. "The diagnostics of a failure cause and the viability of finding a convincing proof." Expert F. "The most difficult part is understanding the problem, because what follows after that is a combination of known procedures."

Expert G: "Finding a cause is a challenge because there are many factors influencing each failure. Sometimes it is also difficult to provide a solution to rehabilitate the facility."

Common features:

- The identification of the cause of failure is seen as the most difficult part in failure analysis.

- Experts coincide that there are many variables in geotechnical failures so that complete certainty regarding causes of failure is seldom achieved.

- Modeling is difficult because of the uncertainty found in geotechnical parameters.

\section{Differences with structural failure analysis}

Another aspect discussed by the experts was the complexity of observing inside a soil mass to understand the nature or extent of a failure. Geotechnical experts highlighted the differences between failures in their field and those faced by structural engineers, who have easier access to all parts of a structure.

The differences between structural and geotechnical failures have been clearly explained by some of the experts interviewed as part of this research. Consider some remarks made by Expert A:

"In geotechnics, what you see is relatively limited in relation to what has occurred beneath the surface. In a structure, in which a column fails, you can observe the column; however, in geotechnics you see only a part, only a face and it is difficult to observe the rest. Then you have to [use your imagination] and because of that, you need to carry out additional explorations. You have to move from 2D to 3D: you can see in two dimensions but you need to imagine [the same thing] in three dimensions".

Finally, geotechnical problems are characterized by experts as being systemic and with uncertainty in the available information. 


\section{Conclusions}

This paper is a first attempt to gather information about the reasoning and methodology employed by experts in the field of geotechnical failure investigations. Not all questionnaires have been completed yet but, from the seven cases already completed (which are $70 \%$ of the target number), it is possible to postulate some trends and common aspects in the responses of experts.

With reference to the first hypothesis considered in this paper, the present interviews indicate that there are two main sources of knowledge that an expert uses when investigating a geotechnical failure: (i) General principles, such as force equilibrium or soil settlement; and (ii) Lessons learned from specific previous cases in which the person participated. The interviews indicate that experts use information from previous cases even though the new case could not be solved by making a complete analogy with previous cases. Similarities tend to be only partial and there are often significant differences (such as differences in size or problem parameters) that severely limit an analogical reasoning. But an important asset in all cases is the previous experience in the specific domain.

Regarding the second hypothesis, in most cases experts formulate a failure hypothesis after the first site visit, and only in a few cases they could explicit a cause of failure before or during the visit. A maturing process seems to be necessary, during which they reconsider the evidence gathered in the field.

The group of experts interviewed seem to work using forward (rather than backward) reasoning: they all thought of a possible cause (which was identified as the most difficult part of the failure analysis) and then proceeded to find evidence in support of that hypothesis. This is consistent with attributes of experts, as reported by Vick ${ }^{8}$.

Within an educational domain, in which there is no easy access to professional cases, it is possible to provide close-to-real experience by means of computer-based simulations. Such approach has been followed to create educational tools in the form of active learning in a simulated environment in the context of structural failure investigations ${ }^{9,10,11}$ and the present study should serve as a basis to implement geotechnical failure investigations. Further, the use of computer-based simulations in an educational environment, as proposed by Schank ${ }^{12}$, include fictitious (simulated) experts to answer questions and tell stories, and those can only be obtained by means of interviews such as those reported in this paper.

Acknowledgements. This work was possible thanks to the support of NSF-CCLI grant DUE0736828: "A Computer-Based Simulated Environment to Learn on Structural Failures in Engineering" (Program Director: Sheryl Sorby). However, the results and opinions expressed are solely of the authors and do not necessarily reflect the views of the funding agency. The authors thank the contributions of the experts who were interviewed and who must remain anonymous. 


\section{References}

[1] Ahmed, S., K.M. Wallace and L.T.M. Blessing, "Understanding the differences between how novices and experienced designers approach design tasks", J. Research in Engineering Design, Vol. 14(1), pp. 1-11, 2003. [2] Petroski, H., Design Paradigms: Case Histories of Error and Judgment in Engineering, Cambridge University Press, 1994.

[3] Delatte, N., Beyond Failure: Forensic case studies for civil engineers, ASCE Press, Reston, VA, 2009.

[4] Kolodner, J., Case-based Reasoning, Morgan Kaufmann, San Mateo, CA, 1993.

[5] Gonzalez, A. J. and D. D. Dankel, The Engineering of Knowledge-Based Systems: Theory and practice, Prentice Hall, Englewood Cliffs, NJ, 1993.

[6] Godoy, L. A., "Interviews with experts, in which they explain how they solved structural failure investigations”, Proc. 2010 ASEE Southeast Section Conference, Blacksburg, VA, April 2010.

[7] Bransford, J.D., A.L. Brown, and R.D. Cocking, How People Learn: Brain, Mind, Experience and School, National Research Council, Washington, DC, 2001.

[8] Vick, S.G., Degrees of Belief: Subjective probability and engineering judgment, ASCE Press, Reston, VA, 2002.

[9] Godoy, L. A., "Learning-by-Doing in a Web-Based Simulated Environment”, Proc. 6th Annual Int. Conf. Information Technology in Higher Education and Training, IEEE, Juan Dolio, Dominican Republic, July 2005. [10] Godoy, L. A., "Developing a Computer-Based Simulated Environment to Learn on Structural Failures", Paper 1849 in: Proc. ASEE Annual Conf., Austin, TX, June 2009.

[11] Godoy, L. A., "Story-centered learning in a computer-based simulated environment", in Proc. ASEE Annual Conf., Louisville, KY, June 2010.

[12] Schank, R. C. and C. Cleary, Engines for Education, Lawrence Erlbaum, Hillsdale, NJ, 1995. 